\title{
LOS EFECTOS DEL SEXISMO NO SE OLVIDAN NI INMEDIATAMENTE NI PERMANENTEMENTE
}

Nuria Arjona Martín y José Miguel García Ramírez. Universidad de Granada.

Fecha de recepción: 30 de junio de 2014

Fecha de revisión: 15 de julio de 2014

Fecha de aceptación: 30 de julio de 2014

\section{Resumen}

Las situaciones de sexismo son un fenómeno social que aparecen en muchas de las situaciones sociales diarias, situaciones que pasan desapercibidas y algunas, mayoritariamente las situaciones de sexismo benévolo, no son percibidas como situaciones sexistas y no cobran la suficiente importancia. El objetivo de este estudio es analizar cómo se relaciona la necesidad de cierre cognitivo frente al sexismo ambivalente. Para ver la relación entre ambas variables se contó con una muestra de 46 mujeres, estudiantes de Educación de la Universidad de Granada, de edades comprendidas entre los 22 y 45 años. Los instrumentos utilizados fueron el Test revisado y adaptado de Necesidad de Cierre Cognitivo (Horcajo, Darío Díaz, Gandarillas y Briñol, 2011) y la Escala de Sexismo Ambivalente (Glick \& Fiske, 1996). Los resultados obtenidos demuestran que a las participantes les causa incertidumbre inmediata y permanente cualquier situación sexista, ya sea hostil o benevolente.

Palabras clave: feminismo, prejuicio, sexismo, cierre cognitivo.

A finales del siglo XIX y durante el siglo XX fue cuando el feminismo tomó especial protagonismo. Esta disconformidad llevo a cuestionar lo que es una mujer, lo que digo lugar a una amenaza sobre el hombre, dando lugar a lo que se conoce como crisis de la masculinidad. El objetivo de la corriente feminista es resolver la desigualdad que sufre las mujeres, siendo estas desigualdades producto de las construcciones socioculturales entendiéndose en un tiempo y lugar determinado. Por lo que el feminismo es un movimiento que surge por la situación de desigualdad entre género. Destacan dos teorías contemporáneas: Feminismo de igualdad, es una postura que tiene a lo masculino como lo ideal, por lo que le propone a las mujeres que persigan los atributos y capacidades que le son concedidos y reanudarlos como propios. Buscando que las mujeres puedan gozar de las mismas oportunidades laborales, sociales y jurídicas que los hombres, aunque sin considerar que el hecho de que se establezcan diferencias sexuales en las relaciones sociales, no hará que se llegue a la igualdad. Y el otro criterio es el feminismo de la diferencia, esta teoría tiene como objetivo el cambio cultural que identifica la posibilidad de establecer nuevas relaciones sociales entre los géneros. En la actualidad, en las sociedades occidentales nos encontramos con un gran cambio en lo que se refiere a la mujer en el espacio privado y público. Las relaciones entre los sexos, a medida, se van adaptando al cambiante entorno económico y social, lo que da lugar a nuevas simbologías que las generaciones siguientes adoptaran como propias, asimilando de manera natural la igualdad de género, como lo era antes la subordinación femenina (Victoria, 2001). 
También las personas han ido cambiando sus actitudes y emociones a la hora de enfrentarse al sexismo como un caso particular de prejuicio. El tono positivo que se le otorga a las relaciones de género se retira de la concepción de prejuicio. Pero si entendemos, prejuicio como una actitud que genera desigualdad, esto nos lleva a convertir al sexismo en uno de los tipos más antiguos de prejuicio. La combinación que caracteriza al sexismo es: afecto más dominancia. Para entender bien el sexismo, hay que tener claro la relación de interdependencia entre los sexos, como los roles tradicionales, atracción heterosexual, dando lugar a la ambivalencia en las actitudes sexistas. (Rodríguez-Bailón, 2012)

Nos encontramos con dos teorías que recogen la dominancia del hombre en todas las culturas:

- Teoría evolucionista, el reflejo de la selección sexual. Los hombres necesitan desarrollar disposiciones competitivas y agresivas para ganar en la competición sexual.

- Teoría socio-estructurales, el reflejo de la evolución societal y las condiciones culturales. Las sociedades basadas en la caza son las más igualitarias. Sin embargo, las sociedades basadas en la agricultura y la industria, surge la competición por recursos y el estatus favorece la estratificación, pero las mujeres han quedado aisladas de los contextos privados debido a su rol en la reproducción, ya que su vida es estar en su casa y cuidar de su familia.

Según la Teoría de los Roles Sociales: "Los estereotipos de género y las diferencias comportamentales entre los sexos provienen de la división de roles y la jerarquización sexual. A los grupos se les asignan rasgos asociados con los roles que desempeñan y la posición social que ocupan." (Eagly \& Koenig, 2006)

Se produce una constante intercultural de dependencia íntima entre hombres y mujeres, institucionalizando el matrimonio y la división tradicional de roles. Por lo que la dominancia se da junto a la interdependencia íntima. Lo que justifica el concepto de sexismo ambivalente que está compuesto por la dominancia masculina (hostilidad) y la interdependencia (ideología de género benevolente). La hostilidad genera la idealización de los hombres que cumplan las funciones de protección y proveedores de recursos, y la benevolencia a la idealización de las mujeres que cubren los objetivos de los hombres, como por ejemplo: esposas, madres, objetos románticos, etc. En la actitud hostil del hombre hacia la mujer, la mujer provoca amenaza a la superioridad del hombre, mientras que la actitud benevolente no provoca tensión ni conflicto. El sexismo ambivalente genera actitudes positivas hacia las mujeres, pero es un tipo de paternalismo, ya que considera a las mujeres como el sexo débil que necesitan ser cuidadas por los hombres. (Garaigordobil \& Aliri, 2011; RodriguezBailon, 2012)

Por lo que surge el concepto de sexismo ambivalente que está formado por dos componentes claramente diferenciados, pero relacionados entre sí -sexismo hostil y el sexismo benévolo-. El sexismo hostil concuerda claramente con el viejo sexismo, sin embargo el sexismo benévolo es un conjunto de actitudes interrelacionadas con las mujeres, que tiene un tono afectivo positivo hacia ellas. El sexismo benévolo es tan perjudicial como el hostil, se puede utilizar para compensar el sexismo hostil, dado que el sexismo hostil no suele considerarse a sí mismo sexista. Los dos sexismos se utilizan para dar el poder estructural al varón. (Glick \& Fiske, 1996; Exposito, Moya \& Glick, 1998; Lameiras \& Rodriguez, 2003, Moyano y col., 2013) 
Las personas necesitan tener ciertos conocimientos que les ayuden a procesar la información y orientar sus conductas, para adaptarse al entorno. Así surge la necesidad de cierre cognitivo, NCC, por la necesidad de buscar y mantener una solución ante un problema determinado, evitando la ambigüedad, confusión y/o incertidumbre. La NCC se compone de dos tendencias complementarias entre sí: la tendencia de urgencia por conseguir un conocimiento determinado, es decir, la tendencia por buscar una solución o respuesta inmediata ante una situación de incertidumbre o problema, y la tendencia de permanencia de ese conocimiento, se refiere al deseo de mantener y perpetuar, lo que da lugar tanto a preservar como a rechazar una nueva información que nos pueda servir para cuestionar o consolidar el cierre cognitivo. Se ha comprobado que la necesidad de cierre cognitivo puede ser aumentada temporalmente manipulando el contexto. Por lo que la NCC será más elevada en situaciones donde sea demasiado costoso o lento el procesamiento de la información. En definitiva, ambas tendencias del cierre cognitivo están apoyadas en la idea de que las personas con una mayor NCC tienden a sufrir la ausencia de una respuesta duradera y clara como algo aversivo, ante situaciones de incertidumbre, por lo que ambas tienden a suprimir ese malestar produciendo el cierre cognitivo permanente y rápido (Chirumbolo y col., 2004; Kruglanski y col., 2006).

\section{Objetivo}

Analizar la correlación entre la necesidad de cierre cognitivo frente al sexismo ambivalente.

\section{Método}

Se trata de un estudio de investigación exploratoria.

\section{Participantes}

Participaron voluntariamente 46 mujeres estudiantes de la Facultad de Ciencias de la Educación de la Universidad de Granada, de edades comprendidas entre los 22 y 45 años.

\section{Instrumentos}

Para mi investigación he utilizado dos tipos de test:

1. Test revisado de Necesidad de Cierre Cognitivo, lo que da lugar a una innovación tanto metodológica como conceptual, ya que mide las dos tendencias promovidas por la necesidad de cierre cognitivo de forma explícita, haciendo algo que no hace otro instrumento de medida de la NCC. En otras palabras, lo novedoso del TR-NCC es que para ello se han diseñado unos items de tal forma que miden explícitamente las dos tendencias causadas por la NCC: la tendencia de urgencia y la tendencia de permanencia. (Horcajo, Darío Díaz, Gandarillas y Briñol, 2011)

2. Escala de Sexismo Ambivalente, ASI, (Glick y Fiske, 1996) con el objetivo de medir el sexismo hostil y benevolente. Los estudios ASI, han demostrado que estos dos componentes del sexismo (hostilidad y benevolencia) son dos componentes del sexismo que están presentes en la mayoría de las culturas, correlacionando positivamente y prediciendo índices de desigualdad de género.

Los resultados obtenidos son analizados a través del programa estadístico SPSS 20, concretamente se les realiza un análisis estadístico de fiabilidad para asegurar que los 
datos obtenidos son fiables. Los análisis de las dos escalas son para la ASI de 0.935 y para la NCC de 0.836. Por lo tanto, ambas escalas muestran un alto grado de fiabilidad, tal como se muestran en las tablas 1 y 2 .

Tabla 1. Estadísticos de fiabilidad de la escala ASI

\begin{tabular}{cc} 
Alfa de Cronbach & $\mathrm{N}$ de elementos \\
\hline, 935 & 22
\end{tabular}

Tabla 2. Estadísticos de fiabilidad de la escala NCC

\begin{tabular}{cc}
\hline Alfa de Cronbach & $\mathrm{N}$ de elementos \\
\hline, 836 & 14 \\
\hline
\end{tabular}

\section{Procedimiento}

La investigación se realiza en la Facultad de Ciencias de la Educación en la Universidad de Granada. Durante la primera semana de mayo, 46 mujeres, estudiantes, rellenaron dos test, la ASI y el NCC. Las participantes son informadas del estudio del que iban a participar y que los datos obtenidos serían recogidos y analizados de forma anónima y confidencial.

Posteriormente los datos obtenidos son analizados por el programa de análisis estadístico SPSS 20.

\section{Resultados}

Los datos obtenidos se analizan a través de un análisis estadístico de correlación de Pearson, cuyos resultados obtenidos muestran que en una situación de sexismo benévolo el cierre cognitivo de urgencia es negativa $\left(-, 518^{* *}\right)$, por lo que no se produce cierre cognitivo ante dicha situación, manteniéndose la incertidumbre o el recuerdo de la situación de sexismo percibida. Ante la misma situación pero teniendo en cuenta el cierre cognitivo de permanencia sigue siendo negativa la correlación $\left(-, 407^{* *}\right)$. Por lo tanto se sigue manteniendo la incertidumbre y recuerdo de la situación sexista percibida. Ante una situación de sexismo hostil podemos observar que el cierre cognitivo de urgencia vuelve a ser negativa $\left(-, 519^{* *}\right)$ igual que en la situación de sexismo benévolo, por lo que no se produce el cierre cognitivo de urgencia frente a una situación de sexismo hostil. En cuanto a la necesidad de cierre cognitivo de permanencia ante una situación hostil aparece con una mayor puntuación negativa (, $\mathbf{5 3 9}^{* *}$ ), por lo tanto, ante la situación de sexismo hostil las participantes mostraron una negativa más alta al cierre cognitivo de permanencia, demostrando que las situaciones sexistas hostiles no se olvidan ni inmediatamente ni permanentemente.

Tabla 3. Análisis de correlaciones de Pearson

NCC urgencia 1

NCC urgencia NCC permanencia ASI benevolente ASI hostil

NCC permanencia, 430 ** $\quad 1$

ASI benevolente,$- 518^{*}$

ASI hostil
1

$-, 407^{* *} \quad 1$

$-, 539^{* *} \quad, 614$
1 


\section{Discusión}

Los resultados obtenidos demuestran que ante una situación de sexismo, cuanto más alto es el sexismo tanto benévolo como hostil tiende a reducirse la necesidad de cierre cognitivo tanto de urgencia como de permanencia. O sea ante ninguna de las situaciones de sexismo, ya sea hostil como benevolente, no se genera la necesidad de cierre cognitivo ni de urgencia ni de permanencia.

El sexismo hostil, es el sexismo antiguo, el "sexismo de siempre", es un sexismo "a plena luz del día", un sexismo manifiesto, por lo que la necesidad de cierre cognitivo frente a este sexismo siempre ha sido negativa, sin embargo, el sexismo benévolo es un sexismo sutil, un sexismo cuya necesidad de cierre cognitivo era alta frente a situaciones de sexismo sutil, ya que estas situaciones no se percibían como machistas. Pero los datos de este estudio de investigación exploratoria demuestran que frente a una situación de sexismo benévolo no existe la necesidad de cierre cognitivo.

Concluyendo, en la actualidad gracias a las reivindicaciones del movimiento feminista, se puede comprobar que las mujeres ya no tienen la necesidad de cerrar cognitivamente ante situaciones de sexismo. El sexismo debe de ser erradicado en una sociedad de igualdad.

\section{Referencias}

Chirumbolo, A., Livi, S., Mannetti, L., Pierro, A., \& Kruglanski, A. W. (2004). Effects of need for closure on creativity in small group interactions. European Journal of Personality, 18(4), 265-278.

Eagly, A.H., \& Koenig, A.M. (2006). Social Role Theory of Sex Differences and Similarities: Implication for Prosocial Behavior. Mahwah: Lawrence Erlbaum Associates Publishers.

Exposito, F., Moya, M., \& Glick, P. (1998). Sexismo ambivalente: medición y correlatos. Ambivalent sexism: Measurement and correlates. Revista de Psicología Social, 13(2), 159-169.

Garaigordobil, M., Aliri, J. (2011). Sexismo hostil y benevolente: relaciones con el autoconcepto, el racismo y la sensibilidad intercultural. Revista de Psicodidáctica/Journal of Psychodidactics, 16(2), 331-350.

Glick, P., \& Fiske, S. (1996). The Ambivalent Sexism Inventory: Differentiating hostile and benevolent sexism. Journal of personality and social psychology, 70(3), 491-512.

Horcajo, J., Díaz, D., Gandarillas, B., \& Briñol, P. (2011). Adaptación al castellano del Test de Necesidad de Cierre Cognitivo. Psicothema, 23(4), 864-870.

Kruglanski, A.W., \& Webster, D.M. (1996). Motivated closing of the mind: "Seizing" and "freezing". Psychological review, 103(2), 263-283.

Kruglanski, A., Pierro, A., Mannetti, L., De Grada, E. (2006). Groups as epistemic providers: need for closure and the unfolding of group-centrism. Psychological review, 113(1), 84-100. 
Lameiras Fernández, M., Rodríguez Castro, Y. (2003). Evaluación del sexismo ambivalente en estudiantes gallegos/as. Acción psicológica, 2(2), 131-136.

Moyano, M., Expósito, F., \& Trujillo, H. M. (2013). Cierre cognitivo, sexismo y religiosidad: diferencias y similitudes entre grupos de adolescentes con distinta cultura. Anales de psicología, 29(2), 501-508.

Rodríguez-Bailón, R. (2012). El poder: su Psicología Social. Revista de Psicología Social, 27 (3), 287-292.

Victoria, S. (2001). Diccionario ideológico feminista. Barcelona: Icaria. 